\title{
Hybrid-chain: An Innovative and Efficient Mixed Blockchain Architecture
}

\author{
Yunpeng Wang ${ }^{1,3}$, Hui Zhao ${ }^{2, *}$, Tao $\mathrm{Li}^{2, *}$, Fan Zhang ${ }^{1}$ and Zhiyong $\mathrm{Li}^{2}$ \\ ${ }^{1}$ College of Computer, Sichuan University, Chengdu, China \\ ${ }^{2}$ College of Cybersecurity, Sichuan University, Chengdu, China \\ ${ }^{3}$ Southwest China Institute of Electronic Technology, Chengdu, China \\ ${ }^{*}$ Corresponding author
}

\begin{abstract}
The blockchain architecture with distributed and decentralized as the main features often face problems such as long transaction confirmation time, low system throughput, and waste of computing resources. These problems are exactly what the traditional centralized architecture can solve. At the same time, in order to improve the distributed consensus efficiency, consensus algorithms such as Proof of stake, delegated proof of stake, proof of space, proof of authority, and PBFT have emerged from Bitcoin's Pow consensus mechanism. These consensus algorithms have their own advantages and Disadvantages. Disadvantages, such as Pos, DPos can improve transaction efficiency, PBFT can improve the fairness of voting, but at the same time POS also bring benefits and voting rights to hold a lot of user tilt issues to a small number of tokens, PBFT brings about a low cost of malicious user attacks and other issues. In order to effectively take advantage of the high security, high reliability of the blockchain, and high efficiency of traditional centralized architectures, and fully utilizing existing consensus mechanisms, this paper presents an innovative hybrid blockchain approach, which mixed the blockchain architecture, Hybrid-chain, consists of the following three type nodes in the architecture: service center nodes responsible for efficient transaction confirmation, dynamic transaction verification nodes participating in transactions, and random verification nodes responsible for transaction fairness verification. On the consensus mechanism, the consensus algorithm selects the corresponding consensus mechanism according to the transaction characteristics. Experiments show that compared with the traditional blockchain architecture, hybrid chain has obvious performance advantages in transaction sending, transaction confirmation and block information storage, which can greatly reduce the transaction sending time by $96 \%$, shorten the confirmation time by $\mathbf{9 5 \%}$, and reduce the number of 79. \% Block information storage space, etc.
\end{abstract}

Keywords_blockchain; hybird-chain; mixed; architecture

\section{INTRODUCTION}

As a decentralized new information system architecture application, blockchain is considered to be a subversive innovation in the computing model following mainframes, personal computers, and the Internet. Its application areas have gradually expanded from financial and virtual currency to smart manufacturing, internet of things, and digital copyright protection [1]. Compared with centered architecture, like the traditional client/server or browser/server model, blockchain architecture mainly uses decentralized consensus mechanisms to maintain blockchain and transactions, making blockchain and transactions unchangeable and traceable, etc., thus, to a large extent, the integrity, security, and availability of blockchain data will be improved; [2][3][4][5][6] respectively described blockchain, related technologies, the summary of smart contracts, etc., shows that there are still many differences in the application of blockchain architecture and centralization. However, compared with the traditional centralized service architecture, the blockchain has the problems of transactional network broadcasting, long confirmation time, low architecture performance, small transaction throughput, miner mining accounting, and waste of resources. [7] pointed out in 2013 that Bitcoin's blockchain has a large impact on global energy, A detailed analysis was conducted in [8][9][10] in 2016 and 2018 respectively.; [11] studied how to avoid resource waste. $[12][13][14][15][16]$ analyzed the efficiency of blockchain in 2016, 2017, and 2018, and compared them with traditional currency transactions, stock transactions, and gold transactions. It is found that virtual tendency transactions represented by Bitcoin are less efficient. The Bitcoin's transactions' confirmation time averaged from 27.24 minutes in 2016, 133.24 minutes in 2017, and up to 959.38 minutes in 2018 (as of February 2, 2018)[19]. Up to now, its largest processing volume occurred on December 25, 2017, and the trading volume was 405,676 per day [20]. Compared to another global payment tool Visa, which can process approximately 24,000 transactions per second [21] and handle 1.5 million transactions per day [22]. [23] reviewed that improving blockchain efficiency and reducing energy consumption is one of the directions for the future of blockchain research. [24] proposes that the efficiency of blockchain's on IoT can be improved by adding user authentication at edge nodes.[25] analyzed and compared R3's Corda, Ethereum Quorum, Hyper ledger Fabric, and Ripple, it was pointed out that the corresponding blockchain and consensus mechanisms are used in the supply chain and healthcare, while others are used in digital identity authentication and digital collections and asset tags.[26] analyzed Ethereum, Hyper ledger Fabric, and IOTA to find a consensus mechanism that can be used for low-power, mobile computing in IoT. [27] pointed out that in the blockchain application, it is necessary to combine the application scenario to select and improve the blockchain to adapt to different business purposes.

In terms of improving efficiency and reducing energy consumption, traditional centralized service architecture has advantages, but centralized applications have such problems as poor transaction traceability and data corruption. Therefore, this paper proposes an innovative Hybrid-chain architecture that 
takes full advantage of the blockchain architecture and the traditional centralized service architecture to establish an efficient, traceable, and tamper-proof new architecture. At the same time, in order to exert the advantages of various consensus mechanisms, the architecture adds a hybrid consensus mechanism algorithm, and can choose the consensus mechanism according to different application scenarios. The second part analyzes the centralized and decentralized service architecture; the third part constructs the Hybrid-chain architecture and model the fourth part is comparison of the traditional blockchain and Hybrid-chain architecture through experiments. Part 5 of this article is summation.

\section{RELATED WORKS}

\section{A. Blockchain Architecture}

The blockchain was derived from the "Point-to-Point Electronic Currency Transactions" published by Satoshi Nakamoto in 2007. The transaction model is mainly used to solve the double- spending problem of digital currency without the need of a trusted third party.[28]. It is a decentralized service architecture composed of a combination of technologies such as $\mathrm{p} 2 \mathrm{p}$ network technology, encryption technology, and consensus mechanism. Its essence is a decentralized distributed database. A block is a data storage unit, and a hash message digest of a block forms a link between the blocks. Finally, each block is linked in a chain structure to form a link. A traceable data link is managed and maintained through a decentralized distributed network. In the consensus mechanism, the blockchain was first solved by the Byzantine fault tolerance. That is, most of the nodes involved in the blockchain confirmed and packaged the transaction to form a block, and the transaction was considered successful. This type of voting mechanism is more suitable for data storage and data protection.

The transactions in the blockchain are initiated by each node participating in the blockchain, and then forwarded in the network; and the confirmation of the transaction is carried out by the "mining" of most miners participating in the blockchain. After successful mining, the transaction within a certain period of time is confirmed and formed into a block [32]; the transmission of the message and its confirmation in the entire blockchain are shown in the Figure I.

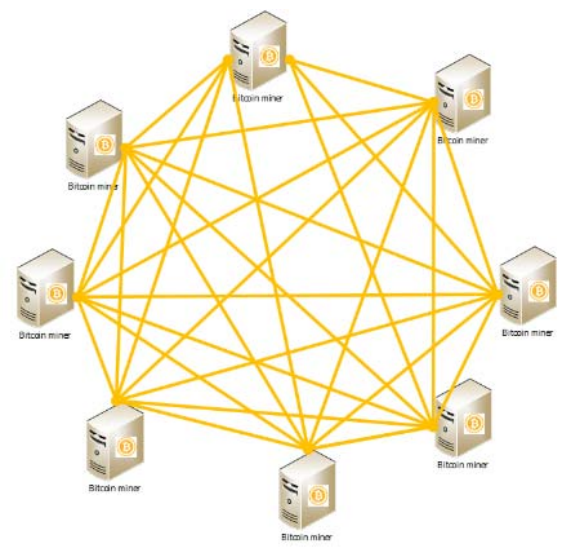

FIGURE I. TRADITIONAL BLOCKCHAIN NETWORK MODEL

\section{B. Centralized Architecture}

The traditional centralized service architecture is commonly used in client-server model and browser/server model. The client-server model is a distributed application structure. It consists of two types of roles: service provider and service requester. The service provider provides resources or services in a centralized manner. The service requester requests the corresponding service resources by accessing the corresponding service provider. . Usually the client and the server run on different hosts, but communicate through the computer network [30]. The browser server architecture is similar to the client/server architecture, and is mainly composed of two types of servers and client browsers [31]. Unlike $\mathrm{C} / \mathrm{S}, \mathrm{B} / \mathrm{S}$ is composed of browser clients.

The central application architecture initiates and confirms messages by the server. After the central server performs transactions, relevant information is displayed to the client and server.

\section{Comparison of the Architecture}

The two architectures are different in terms of transaction sending, transaction confirmation, and information storage. Two architectures with $\mathrm{n}$ nodes are used to compare one piece of message, as shown in Table I.

TABLE I. COMPARSION OF SENDING, CONFIRMATION\&STORAGE

\begin{tabular}{|c|c|c|}
\hline $\begin{array}{c}\text { Architecture } \\
\text { type }\end{array}$ & $\begin{array}{c}\text { Blockchain } \\
\text { Architecture }\end{array}$ & $\begin{array}{c}\text { Central } \\
\text { Application }\end{array}$ \\
\hline $\begin{array}{c}\text { Whether Send to } \\
\text { other Nodes }\end{array}$ & YES & NO \\
\hline Ssend Times & Client count & 1 \\
\hline $\begin{array}{c}\text { Whether Need Other } \\
\text { Nodes' Confirmation }\end{array}$ & YES & NO \\
\hline Confirmation & YES & No \\
\hline Confirmation Times & Client Count & 1 \\
\hline $\begin{array}{c}\text { Wheter Need Distributed } \\
\text { Storage }\end{array}$ & YES & NO \\
\hline Storage Type & Decentralized & Centralized \\
\hline Storage Times & Client Count & 1 \\
\hline
\end{tabular}

Comparison of the blockchian and central application's related functions is shown in Table II.

TABLE II. COMPARISON OF KEY FEATURES

\begin{tabular}{|c|c|c|}
\hline Architecture & $\begin{array}{c}\text { Blockchain } \\
\text { Architecture }\end{array}$ & $\begin{array}{c}\text { Central } \\
\text { Application }\end{array}$ \\
\hline Comparisons & & NO \\
\hline Traceable & YES & NO \\
\hline Delivery Time & YES & Short \\
\hline Confirmation Time & To long & Short \\
\hline Storage Time & To long & Short \\
\hline Safety & High & Relatively Low \\
\hline
\end{tabular}




\section{HYBRID-CHAIN ARCHITECTURE MODEL}

\section{A. Hybrid-chain Architecture Design}

The Hybrid-chain centralized blockchain architecture takes full advantage of the blockchain architecture and the features of a centralized application. The design includes a cluster centralized billing service node, a group of transaction validation client nodes and a set of random proof nodes: the centralized billing service nodes consists of one or more center nodes. The nodes can be composed of a traditional centralized service architecture or a decentralized blockchain architecture. The centralized billing service nodes confirms, records, and distributes all transactions. The transaction verification node consists of blocks. The parties involved in the transactions include the transaction initiator and the transaction receiver. The transaction verification client nodes changes with the constant changes of the transaction initiator and the transaction receiver in the block. That is, the transaction verification node only records and verifies that is related to its own transaction. Block information and transactions that are not related to them are not recorded and verified. All the block verification nodes perform traceability of the verification node through the previous block verification node list and the next block verification node list, and all block information is passed through the previous block information hash and the next block information hash. The random proof nodes is randomly generated in the nodes where no transaction occurs, the architecture of Hybrid-chain is shown in Figure II.

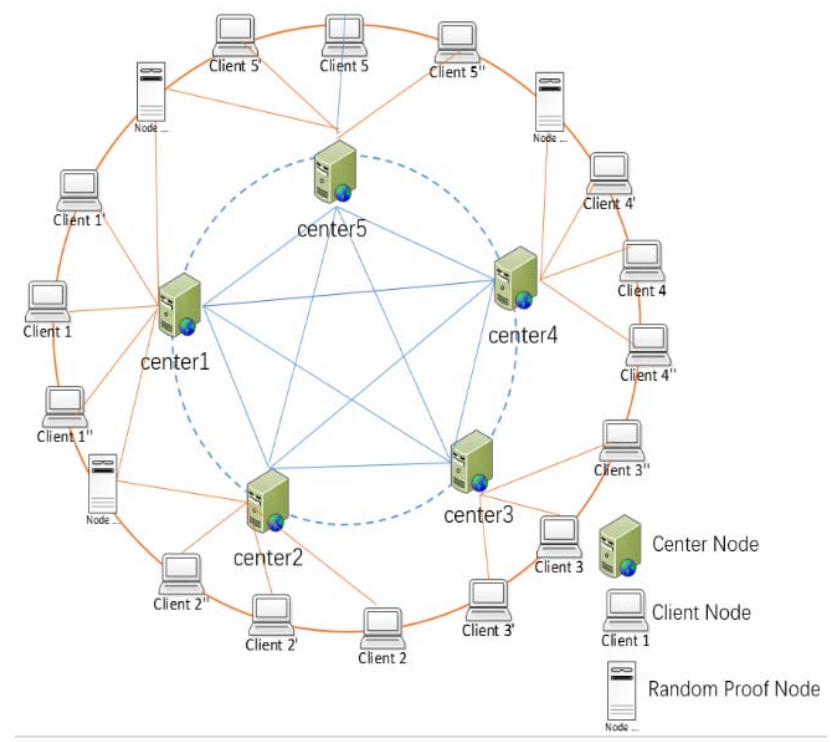

FIGURE II. THE ARCHITECTURE OF HYBRID-CHAIN

\section{B. Consensus Mechanism Design}

The consensus mechanism of the traditional blockchain include Proof of work, Proof of stake, delegated proof of stake, proof of space, proof of authority and so on.[29] In the Hybridchain, all the traditional blockchain's consensus mechanisms can be used. The consensus mechanism has its own advantages and some drawbacks. For example, the POW mechanism has good openness, but the use of the POW consensus mechanism on a large scale will cause waste of resources. Using the POS working mechanism is more efficient, but it will cause the rights and voting rights to be held by a small number of tokens. PBFT fairness is better, but malicious users attack low cost.

In the Hybrid-chain, the central server is only a small cluster, so we can use the POW mechanism to make sure the architecture more openness, and in the verification nodes we can use the POS to improve efficiency. Then at the runtime of the Hybrid-chain, there are more than one sub blockchain or more than one consensus mechanism is running, the blockchains are mixed and the consensus are mixed. The Figure III shows that the blockchain Main is formed with the service center nodes, a dynamic transaction verification node constitutes blockchain A, and other transactions constitutes blockchain A, blockchain B and blockchain $\mathrm{C}$.

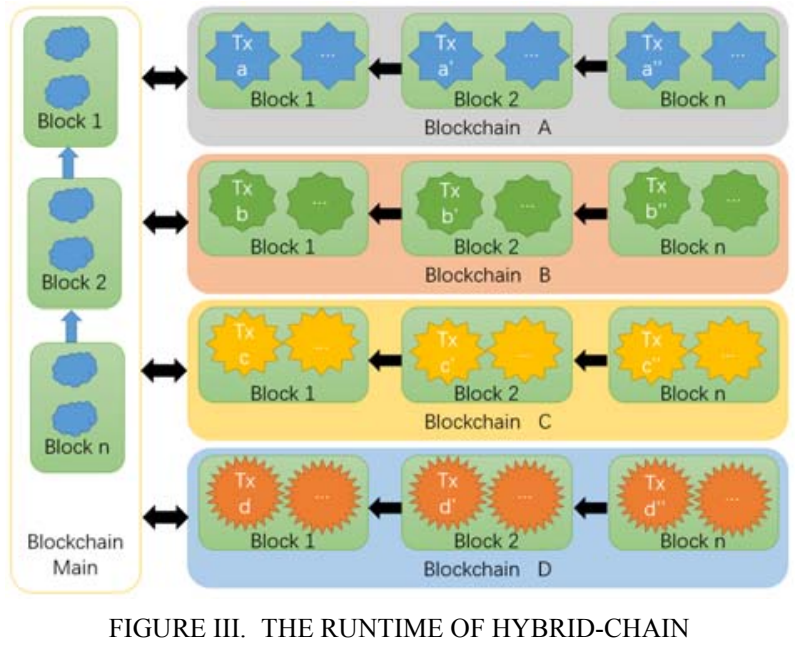

\section{Transaction Delivery and Verification}

In the blockchain, if user A sends a transaction to user B, firstly, the node in which $\mathrm{A}$ is located sends transaction information to all other nodes connected to it, these nodes verify the transaction after receiving the transaction. If the verifications passed, these nodes forward the transaction to other nodes connected to it. A blockchain containing n nodes, the transaction ultimately needs to be verified and sent through n nodes;

In the centralized service architecture, if user A sends a transaction to user $\mathrm{B}$, the process is that user a makes a transaction request to the center server via its client or browser, and the center server verifies the transaction. After the verification is passed, the server sends the corresponding notification information to the client or browser where user B is located.

For the Hybrid-chain architecture as shown in Figure IV., when the user A sends a transaction to the user B, the node where the user A is located sends the transaction to the centralized accounting service node, which will verify this transaction firstly, if the verification is passed, then the central billing service node sends the transaction to other nodes that are currently involved in the transaction for verification. As shown in the above figure, if the current transaction involves client1,client1', client1', the confirmation of the transaction only requires the central service node, client1,client1', client1" to verify; and if the related transaction of the next block is related to client2, client2', client2', client3, client3', then the central service node, client2, 
client2', client2',client3, client3' are required to verify the transaction, and Table III. Compares the transmission of the three transactions in the nodes:

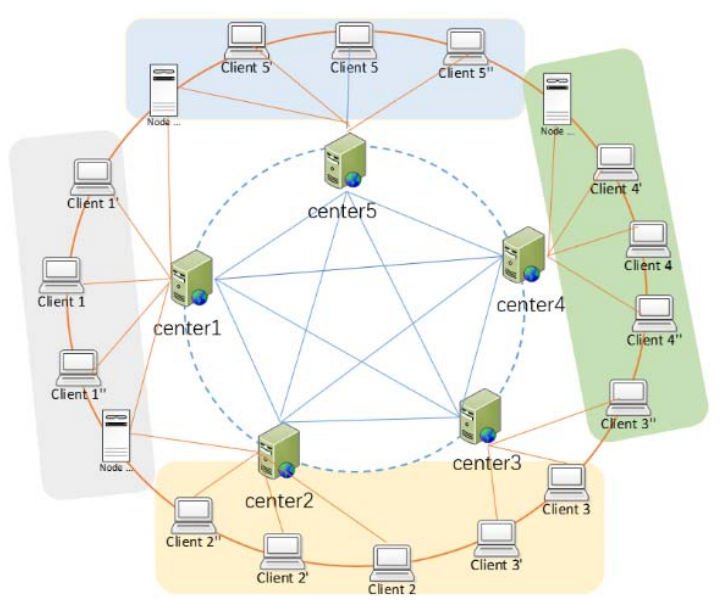

FIGURE IV. EXAMPLE OF A TRANSCATION DELIVERY AND VERIFICATOIN

TABLE III. COMPARISON OF THE TRANSMISSION

\begin{tabular}{|c|c|c|c|}
\hline $\begin{array}{c}\text { Architecture } \\
\text { type }\end{array}$ & $\begin{array}{c}\text { Need to } \\
\text { send }\end{array}$ & $\begin{array}{c}\text { The } \\
\text { minimum to } \\
\text { be sent }\end{array}$ & $\begin{array}{c}\text { The maximum } \\
\text { to be sent }\end{array}$ \\
\hline $\begin{array}{c}\text { Blockchain } \\
\text { architecture }\end{array}$ & yes & $\mathrm{n}$ & $\mathrm{n}$ \\
\hline $\begin{array}{c}\text { Central } \\
\text { Application }\end{array}$ & no & 1 & 1 \\
\hline Hybrid-chain & yes & 3 & $\mathrm{n}$ \\
\hline
\end{tabular}

The Hybrid-chain satisfies formula (1) when the transaction is sent and confirmed, the min send times is 3 ,and the max send times is $\mathrm{n}$, at other times, the send times is decided by the center server numbers, the random poof nodes and the client attended to the transactions.

$$
\left\{\begin{array}{c}
f(\text { send })_{\min }=3 \\
f(\text { send })_{\max }=n \\
f(\text { send })_{\text {other }}=a_{\text {servers }} n+b_{\text {proof }}+c_{\text {validate }} n
\end{array}\right.
$$

The blockchain satisfies the requirement of formula (2) on the number of transactions sent, the max send time is decided by the cluster of the blockchain, like the bitcoin, the cluster of the blockchain is 8 .

$$
\left\{\begin{array}{c}
f(\text { send })_{\min }=0.51 * n \\
f(\text { send })_{\max }=n * d_{\text {cluster }}
\end{array}\right.
$$

Let assume that:

$$
\begin{aligned}
& a_{\text {servers }}=0.1 \\
& c_{\text {validate }}=0.1 \\
& b_{\text {proof }}=50
\end{aligned}
$$

The number of transaction send and confirmed compared blockchain and Hybrid-chain is show in Figure V. As the number of nodes increases, the difference between the number of nodes required by the two architectures increases.

Taking the Hybrid-chain shown in Figure IV. Assume that the blockchain is also composed of $n$ nodes. In each of the $n$ nodes blockchian or Hybrid-chain, 4 transactions are sent, the node relationship is shown in Table IV.

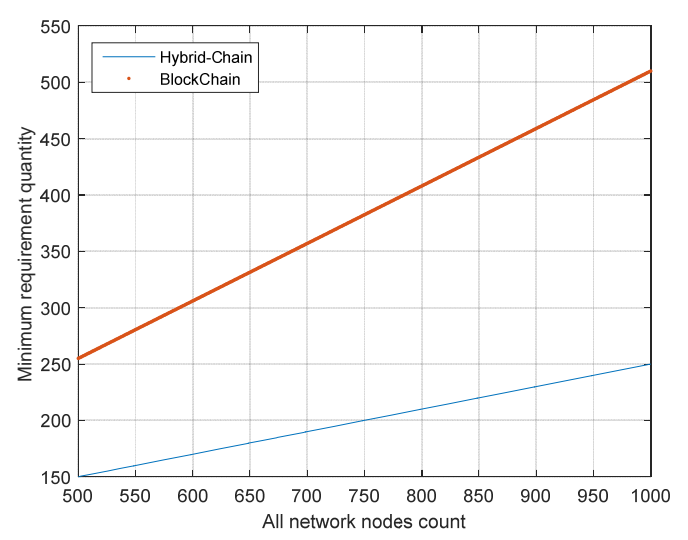

FIGURE V. EXAMPLE OF SERVICE CENTER NODES'S BLOCK CONTENT

TABLE IV. COMPARISON OF THE TRANSACTION VERIFICATION

\begin{tabular}{|c|c|c|}
\hline $\begin{array}{c}\text { Transaction } \\
\text { No. }\end{array}$ & Blockchain & Hybrid-chain \\
\hline 1 & $\begin{array}{c}\text { all attend } \\
\text { nodes }\end{array}$ & $\begin{array}{c}\text { central Server, } \\
\text { client1,client1', client 1', } \\
\text { random proof nodes }\end{array}$ \\
\hline 2 & $\begin{array}{c}\text { all attend } \\
\text { nodes } \\
\text { central Server, } \\
\text { client2, client2', client2',client3, } \\
\text { client3' } \\
\text { random proof nodes }\end{array}$ \\
\hline 3 & $\begin{array}{c}\text { all attend } \\
\text { nodes }\end{array}$ & $\begin{array}{c}\text { central Server, }, \text {, client 4',client 4',client 4 } \\
\text { random proof nodes }\end{array}$ \\
\hline 4 & $\begin{array}{c}\text { all attend } \\
\text { nodes }\end{array}$ & $\begin{array}{c}\text { central Server, } \\
\text { Client 5,client 5',client 5', } \\
\text { random proof nodes }\end{array}$ \\
\hline
\end{tabular}

\section{Transcation Confirmation}

After the node in the blockchain receives the transaction, it needs to verify and confirm the transaction. Take Bitcoin as an example, the process of transaction confirmation is mainly the package verification of the POW workload proof mechanism implemented by the miners participating in the Bitcoin blockchain.

In the centralized service architecture, the confirmation of the transaction occurs in the central server, and the server stores the information of both parties of the transaction.

When the Hybrid-chain architecture confirms the transaction, the central billing service node sends the transaction to the transaction-related party, and the transaction-related party confirms the transaction. After both parties confirmation, the confirmation result is sent to the central service billing node. The central service billing node packages the transaction. 
As an example of the Hybrid-chain of a block chain composed of 7 segments in transaction transmission, the confirmation of the transaction of the blockchain requires the confirmation of all nodes greater than $51 \%$ of the clients: client 1 , client2, client3, client4, client5, client6, and client7. The Hybridchain requires the central server and two other participating clients to confirm.

The node Hybrid-chain required for transaction confirmation is formula (3), and blockchain is formula (4):

$$
\left\{\begin{array}{c}
f(\text { confirm })_{\min }=3 ; \\
f(\text { confirm })_{\max }=n ; \\
f(\text { confirm })_{\text {other }}=a_{\text {sercers }} n+b_{\text {proof }}+c_{\text {validate }}
\end{array}\right.
$$

The blockchain meets the formula's requirements for the number of transactions sent:

$$
\left\{\begin{array}{c}
f(\text { confirm })_{\min }=0.51 * n \\
f(\text { confirm })_{\max }=n^{*} d_{\text {cluster }}
\end{array}\right.
$$

\section{E. Block Storage}

In blockchain architecture, transactions are stored at each miner node. Bitcoin blocks are stored in miner stores around the world, and blocks are stored in the Bitcoin core client.

When the Hybrid-chain performs block storage, the center server performs full storage of block data, which blocks contain all blockchain's block hash information, the schematic diagram shown in Figure VI, and each node stores only the upstream and downstream relationships of the blocks and blocks related to its own transactions, taking the scenario in block transmission as an example. Each block stores only two transactions. The comparison between blockchain and Hybrid-chain on block storage is shown in Table V.

TABLE V. COMPARISON OF THE BLOCK STORAGE

\begin{tabular}{|c|c|c|}
\hline $\begin{array}{c}\text { Block } \\
\text { No. }\end{array}$ & Blockchain & Hybrid-chain \\
\hline 1 & $\begin{array}{c}\text { all attend } \\
\text { nodes }\end{array}$ & $\begin{array}{c}\text { central Server, } \\
\text { client1,client1', client1', }\end{array}$ \\
\hline 2 & $\begin{array}{c}\text { all attend } \\
\text { nodes } \\
\text { central Server, } \\
\text { client2, client2', } \\
\text { client2',client3, client3, }\end{array}$ \\
\hline 3 & $\begin{array}{c}\text { all attend } \\
\text { nodes } \\
\text { central Server, } \\
\text { client3 ', client 4',client 4",client } \\
4\end{array}$ \\
\hline 4 & $\begin{array}{c}\text { all attend } \\
\text { nodes }\end{array}$ & $\begin{array}{c}\text { central Server, } \\
\text { Client 5,client 5',client 5, }\end{array}$ \\
\hline
\end{tabular}

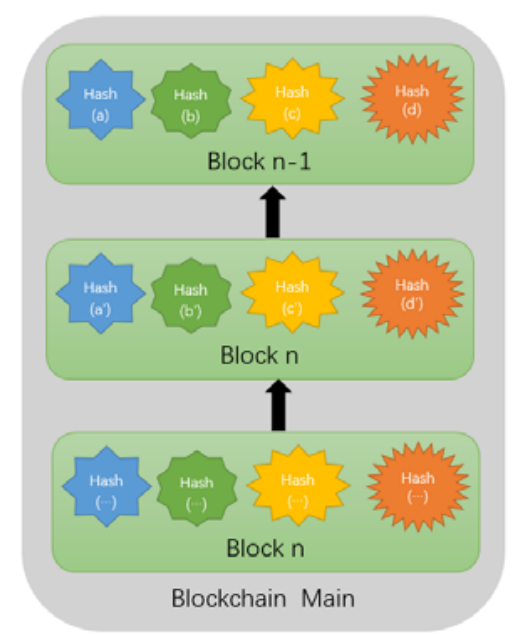

FIGURE VI. EXAMPLE OF SERVICE CENTER NODES'S BLOCK CONTENT

\section{EXPERIMENTS}

\section{A. Prerequisites}

In the experimental stage, the steps of transaction initiation, transaction transmission, block confirmation, and block synchronization are simulated in the real-time environment of the blockchain and Hybrid-chain architectures, respectively, to enhance the authenticity of the experiment in the experiment. In the process of setting up the scene, these simulations will be performed on the test with reference to the real environment of Bitcoin. These descriptions will be based on the assumptions:

1) Suppose a piece of transactions information containing information header and information body. Specific models include Transaction, Transaction_inputs, and Transaction_outputs. Each transaction information is 800 bytes in capacity. The specific model is as follows:

Transactoin (TransactionID, BlockID, TransactionHash, TransactionVersion, TransactionLockTime)

Transaction Inputs (TransacitonInputId, TransacitonID, SourceTransactionOutputID, SourceTransactionHash, TransactionOutputIndex)

Transcation_Outputs (TransacitonOutputID, TransactionID, OutputIndex, OutputValue, OutputScript)

2) In accordance with hypothetical block, the block contains the basic information of the block header and the block body, the block header store the basic information of the block, and the basic model is Block. The size of the block is about 1.1MB, the size of the block header is 500Bytes, and each block can contain up to 1250 transactions.

Block (BlockID, BlockHash, PreviousBlockHash, BlockTimeStamp)

3) The current blockchain confirmation and packing time is not fixed, and there is a certain degree of randomness. Bitcoin, for example, uses the Pow consensus mechanism to compute and package the transactions. Its packaging time is controlled by the transaction calculation difficulty system. Since 2008 , the block packaging time is about 10 minutes, in order to facilitate the 
testing of blockchain and Hybrid-chain's throughput and reduce the randomness brought by Pow, this experiment assumes that Pow difficulty is very low, the times of the computing the information is one, and each block is based on the number of transactions for packaging, when the number of transactions reaches 1250 , the block is packaged;

4) Both the blockchain and the Hybrid-chain are edgeconnected via $\mathrm{p} 2 \mathrm{p}$. The number of neighboring nodes per node is 8. Assume that the time delay for each node to receive messages and verify messages is $0.01 \mathrm{~S}$;

\section{B. Experiment Scenario Design}

When simulating blockchain and Hybrid-chain, we chose the more commonly used $\mathrm{p} 2 \mathrm{p}$ network application simulation tool p2pSim. The current Bitcoin miners in the world is about 6000 , then we choose 6000 is the number of the blockchain and Hybrid-chain nodes. The initiator of the transaction and the receiver of the transaction are randomly selected in the $\mathrm{p} 2 \mathrm{pSim}$ pool. The experiments includes the following two types: singletype transaction experiment and multiple type transaction experiment.

1) Single-type transaction experiment design: 10,000 transactions will be sent to the blockchain and Hybrid-chain respectively. The transaction's size is $1 \mathrm{~KB}$.

2) Multiple type transaction experiment design: the different size transactions will be send in this type of experiment, the size and the number of the transaction is shown in Table VI.

TABLE VI. MULTIPLE TRANSACTIONS EXPERIMENT DESIGN

\begin{tabular}{|c|c|c|}
\hline $\begin{array}{c}\text { Transaction } \\
\text { type. }\end{array}$ & $\begin{array}{c}\text { Transaction } \\
\text { Size. }\end{array}$ & Number of transactions \\
\hline Small & $1 \mathrm{~KB}$ & 10000 \\
\hline medium & $1 \mathrm{MB}$ & 1000 \\
\hline bigger & $100 \mathrm{MB}$ & 1000 \\
\hline
\end{tabular}

\section{Experiment Results}

The single-type transaction experiment by sending 10,000 transactions to the blockchain and Hybrid-chain simulators, the comparison between the two in terms of transaction delivery and transaction confirmation is shown in Figure VI, the time used of Hybrid-chain is about 18 seconds, and the blockchain's used time is about 500 seconds, we conclude that the Hybrid-chain's is more efficient than the block chain in the experiment.

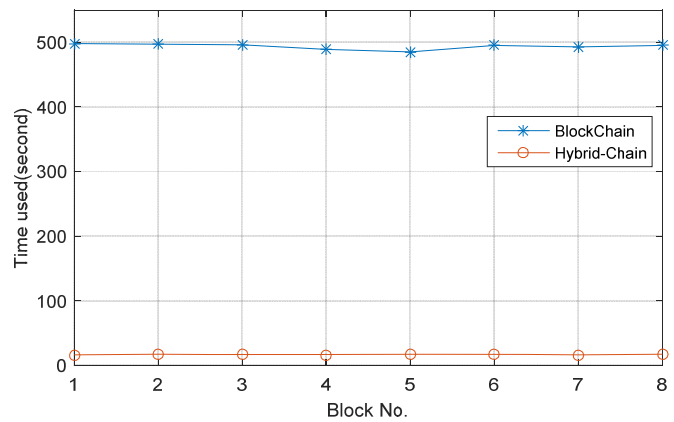

FIGURE VII. THE TIME USED FOR SENDING 1250 TRANSACTIONS COMPARED BLOCKCHAIN WITH HYBRID-CHAIN
The use of two architectures for block synchronization is shown in Figure VII., the Hybrid-chain's block synchronization time is about 2 seconds, and the blockchain's time is about 30 seconds.

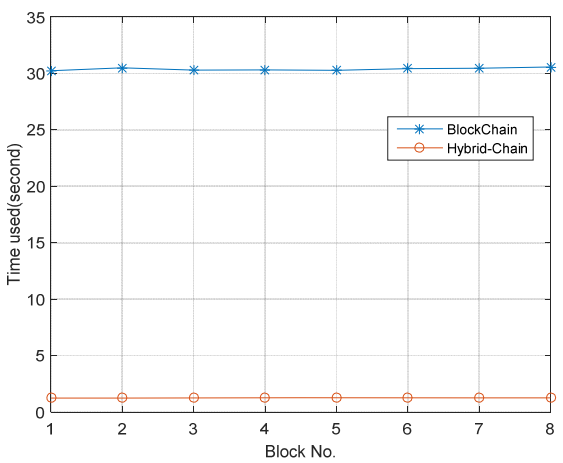

FIGURE VIII. THE TIME USED FOR A BLOCK SYNCH BETWEEN NODES COMPARED BLOCKCHAIN AND CHENTRAL-CHAIN

The space occupied by the blockchain in all nodes is 52800 $\mathrm{MB}$, and the space used by the Hybrid-chain point is $11003 \mathrm{MB}$, which has more space than the blockchain.

The Multiple type transaction experiment by sending different size and number transactions, the time used is show in Figure IX.

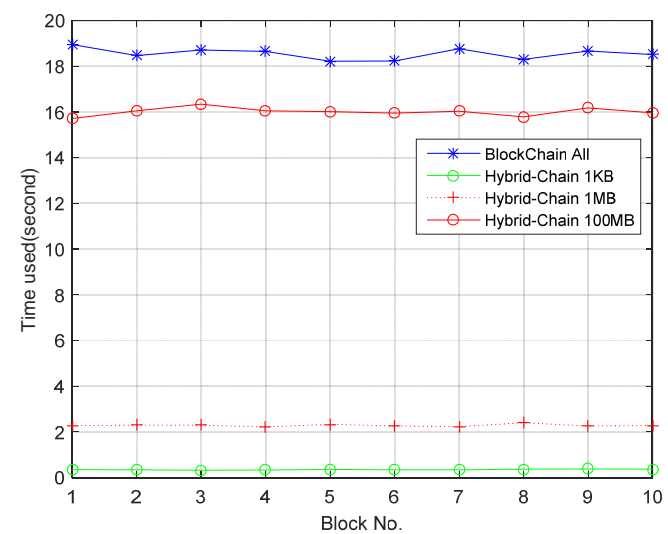

FIGURE IX. THE TIME USED FOR SENDING 1250 TRANSACTIONS COMPARED BLOCKCHAIN WITH HYBRID-CHAIN

Through the above comparison, the Hybrid-chain has greater performance improvement than blockchain in terms of transaction sending, transaction confirmation, and block synchronization, and it occupies less space in block storage.

\section{CONCLUSION}

This Hybrid-chain combines the advantages of blockchain architecture and traditional centralized application architecture to propose an innovative Hybrid-chain model. Through the analysis of the Hybrid-chain architecture in terms of transaction transmission and block synchronization, it is found that Hybridchain has higher efficiency in the transaction then traditional blockchain. By using the different distributed consensus mechanism, the hybrid-chain can used for different application 
fields. Experiment shows the time-consuming links of traditional blockchain, such as sending, transaction confirmation, and block synchronization, have greater performance improvements. Combined with the Hybrid-chain transaction verification node, it is possible to trace transactions and prevent tampering with transactions.

\section{ACKNOWLEDGEMENT}

This work is supported by National Key Research and Development Program of China (Grant No. 2016YFB0800600) Natural Science Foundation of China (Grant No. U1736212 and No. 61572334) and Sichuan Province Key Research and Development Project of China (Grant No. 2018GZ0183) and the Fundamental Research Funds for the Central Universities (No.YJ201727)

\section{REFERENCES}

[1] Swan M Blockchain: Blueprint for a New Economy.O'Reilly Media Inc.; 2015 .

[2] Garay J, Kiayias A, Leonardos N. The bitcoin backbone protocol:analysis and applications. Annual International Conference on the Theory and Applications of Cryptographic Techniques.Springer; 2015:281-310.

[3] Mikołajewicz-Wo zniak A, Scheibe A: Virtual currency schemes the future of financial services. Foresight 2015, 17:365-377.

[4] Zohar A.Bitcoin: under the hood. Commun ACM 2015, 58:104-113.

[5] Tschorsch F, Scheuermann B. Bitcoin and beyond: a technical survey on decentralized digital currencies. IEEE Commun SurvTutor 2016, 18:2084-2123

[6] Diedrich, H. Ethereum: Blockchains, digital assets,smart contracts, decentralized autonomous organizations. Wildfire Publishing.2016

[7] Esmaeilzadeh H, Blem E, Amant RS, Sankaralingam K, Burger D.Powe challenges may end the multicore era. Commun ACM 2013, 56-:93-102

[8] Harwick C.Cryptocurrency and the problem of intermediation.Independ Rev 2016, 20:569-588

[9] Digiconomist. Bitcoin Energy Consumption Index. 2018.

[10] Alex de Vries.Bitcoin's Growing Energy Problem.Joule.2018.

[11] Sara Saberi, Mahtab Kouhizadeh, Joseph Sarkis.Blockchain technology: A panacea or pariah for resources conservation and recycling?.Resources, Conservation \& Recycling.Elsevier.2018

[12] Urquhart, A., 2016. The inefficiency of Bitcoin. Econ. Lett. 148, 80-82.

[13] Bariviera, A.F., 2017. The inefficiency of Bitcoin revisited: a dynamic approach. Econom. Lett. 161, 1-4.

[14] Ahmet Sensoy.The inefficiency of Bitcoin revisited: A high-frequency analysis with alternative currencies.Finance Research Letters.2018.

[15] Ladislav Kristoufek.On Bitcoin markets (in)efficiency and its evolution.Physica A 503 (2018) 257-262.Elsevier.

[16] Aviral Kumar Tiwari, R.K. Jana, Debojyoti Das, David Roubaud. Informational efficiency of Bitcoin-An extension.Economics Letters. 2018

[17] Khamis Hamed Al-Yahyaee, Walid Mensi,Seong-Min Yoon.Efficiency, multifractality, and the long-memory property of the Bitcoin market: A comparative analysis with stock, currency, and gold markets.Finance Research Letters.2018

[18] P.G. Lopez, A. Montresor, D. Epema, A. Datta, T. Higashino, A. Iamnitchi, M.Barcellos, P. Felber, E. Riviere, Edge-centric computing: Vision and challenges,SIGCOMM Comput. Commun. Rev. 45 (2015) $37-42$.

[19] BLOCKCHAIN. IO. Average transaction confirmation time https://blockchain.info/charts/avg-confirmation-time, Accessed: 2018$05-15$

[20] blockchain.info, Confirmed transactions per day, 2017. URL https://blockchain.info/charts/n-transactions?timespan=all/\#
[21] Bitcoin and Ethereum vs Visa and PayPal - Transactions per second.http://www.altcointoday.com/bitcoin-ethereum-vs-visa-paypaltransactions-per-second/, Accessed 2018-05-15

[22] Accept Visa Payments .https://usa.visa.com/run-your-business/acceptvisa-payments.html, Accessed 2018-05-15

[23] JesseYli-Huumo,DeokyoonKo,SujinChoi*, where Is Current Research on Blockchain Technology?-A Systematic Review .Plos one.2016

[24] Zulfiqar Ali,M. Shamim Hossain,Ghulam Muhammad,Ihsan Ullah,Hamid Abachi, Atif Alamri.Edge-centric multimodal authentication system using encrypted biometric templates.Future Generation Computer Systems.2018

[25] Melanie Swan.Blockchain for Business: Next-Generation Enterprise Artificial Intelligence Systems.Advances in Computers.4 May 2018.

[26] Matevž Pustišek,Andrej Kos.Approaches to Front-End IoT Application Development for the Ethereum Blockchain.Procedia Computer Science, Volume 129, 2018, Pages 410-419

[27] Bruno Rodrigues,Thomas Bocek,Burkhard Stiller.The Use of Blockchains: Application-Driven Analysis of Applicability.Advances in Computers,2 May 2018.

[28] Nakamoto, Bitcoin: A Peer-to-Peer Electronic Cash System. Satoshi Nakamoto, 2008.10.08

[29] .Decker, C., Wattenhofer, R.: Information propagation in the Bitcoin network. In: IEEE Thirteenth International Conference on Peer-to-Peer Computing (P2P), pp. 1-10 (2013)

[30] Client-server-model. https://en.wikipedia.org/wiki/Client\%E2\%80\%93server model, Accessed 2018-05-15

[31] Request-response. https://en.wikipedia.org/wiki/Request $\%$ E2\%80\%93response,Accessed 2018-05-15

[32] Melanie Swan.Blockchain for Business: Next-Generation Enterprise Artificial Intelligence Systems.Advances in Computers.4 May 2018. 